\title{
Federation Lifecycle Management Incorporating Coordination of Bio-inspired Self-management Processes
}

\author{
Brian Meskill • Sasitharan Balsubramaniam • \\ Rob Brennan · Kevin Feeney • \\ Brendan Jennings
}

Received: date / Accepted: date

\begin{abstract}
As it has evolved, the Internet has had to support a broadening range of networking technologies, business models and user interaction modes. Researchers and industry practitioners have realised that this trend necessitates a fundamental rethinking of approaches to network and service management. This has spurred significant research efforts towards developing autonomic network management solutions incorporating distributed self-management processes inspired by biological systems. Whilst significant advances have been made, most solutions focus on management of single network domains and the optimisation of specific management or control processes therein. In this paper we argue that a networking infrastructure providing a myriad of loosely coupled services must inherently support federation of network domains and facilitate coordination of the operation of various management processes for mutual benefit. To this end, we outline a framework for federated management that facilitates the coordination of the behaviour of bio-inspired management processes. Using a case study relating to distribution of IPTV content, we describe how Federal Relationship Managers realising our layered model of management federations can communicate to manage service provision across multiple application/storage/network providers. We outline an illustrative example in which storage providers are dynamically added to a federation to accommodate demand spikes, with appropriate content being migrated to those providers servers under control of a bio-inspired replication process.
\end{abstract}

Keywords Network Management · Federation · Bio-inspired Processes · IPTV Content Distribution

Brian Meskill · Sasitharan Balasubramaniam · Brendan Jennings

TSSG, Waterford Institute of Technology, Cork Rd., Waterford, Ireland

E-mail: bmeskill@tssg.org, sasib@tssg.org, bjennings@ieee.org

Rob Brennan · Kevin Feeney

KDEG, School of Computer Science and Statistics, Trinity College Dublin, Dublin 2, Ireland E-mail: rob.brennan@cs.tcd.ie, kevin.feeney@cs.tcd.ie 


\section{Introduction}

The Internet was originally developed for a socio-technological environment that was very different from that of today - small numbers of stakeholders, institutional membership, not for profit services, largely static data traffic behaviour, small numbers of connected devices and at best continental reach. However, as society at large has grasped the potential of the Internet, users are straining the limits of what today's technologies can deliver. This uptake is largely fueled by a proliferation of new and sophisticated services, such as IPTV, that are delivered to a diverse set of increasingly mobile devices in a wide variety of dynamic organisational contexts and which, due to their loosely coupled nature, can be combined in novel ways. Since we believe network and service management is, at its heart, a resource allocation problem, recent dramatic increase in the dynamism, demands, constraints and heterogeneity have made effective solutions very difficult to find.

In the face of these dynamic requirements, Future Internet researchers (e.g. FIND, GENI, AKARI [31]) have been working towards new solutions that can be embedded into the network to support more adaptable and efficient communication. One avenue of research that has attracted particular attention in recent years, is autonomic communications $[9,12,16,23]$. The aim of autonomic communications is to allow networks to have the ability to self-govern within the constraints of business goals that are specified by humans. Through autonomic network communications, more flexible management can be achieved to enable systems to adapt to environmental or organisational changes, with minimal impact on operational expenditure. Autonomic systems are inspired by the human autonomic system that sub-consciously controls many parts of our anatomy, like the beating of our hearts. Closely related is the concept of biologically inspired networking, based on a broader set of processes modelled on biological systems, processes that often display a tremendous ability to adapt as individuals or groups in response to environmental change, e.g. [37,38].

The bulk of research on autonomic networks to date has focused on single network domains and specific management or control applications such as load balancing. However, the Internet incorporates not only a complex patchwork of heterogeneous network technologies, but also a complex patchwork of heterogeneous management domains and specific management applications. We argue, therefore, that mechanisms for federation between management domains and pipelining of diverse management applications will be required, alongside dynamic, self-managing, embedded network processes. We rely on biological mechanisms as a source of inspiration for autonomic network management processes, but we take this one step further. In the same way that autonomic network research to date has only focused on single domains, the majority of bio-inspired network solutions have only focused on a single biological process that solves one specific problem (e.g. routing, resource management) in isolation. Since the evolving Internet will witness an increasing number of heterogeneous networks that must inter-operate in an efficient, robust and scalable manner, new solutions are required to allow multiple autonomic bio-inspired management processes to co-exist together across multiple management domains in the presence of organisational dynamism. This paper focuses on the core requirements for support network federation with this degree of flexibility and outlines our federation architecture, which is designed to satisfy these requirements. We illustrate our approach via a case study involving support for dynamic organisational change and secure resource allocation in a virtual IPTV service provider and its partner, a storage reseller. 
Table 1 Biological processes that have served as inspiration for novel approaches to various network management problems.

\begin{tabular}{ll}
\hline Network Management Problem & Biological Process \\
\hline Routing & Swarm Intelligence (eg. ant colony be- \\
& hviour) \\
Network security & Immune systems \\
Wireless device cooperation & Quorum sensing, chemotaxis, reaction- \\
& diffusion, firefly synchronisation \\
Resource management & Homeostasis equillibrium process, symbio- \\
& sis \\
Network service lifecycle management & Evolution, embryonic models \\
Content and file management & Cell development and signalling processes, \\
& epidemic spreading \\
\hline
\end{tabular}

\section{Federation of Systems Employing Bio-inpired Processes}

Over the last decade, networking researchers have increasingly turned towards biology for inspiration when developing more efficient, scalable and robust communications networks $[13,26,29]$. Table 1 illustrates some key examples of communication network management problems that have addressed using approaches inspired by biological systems.

Designing a system with multiple biological processes is a major challenge, and to date has not been fully investigated by the research community. The main problem is the need to ensure that multiple bio-inspired processes are compatible and co-exist without leading to any conflicts, as they can in nature. Integrated bio-inspired systems and processes exist at various levels, and can be categorised in a hierarchical manner as illustrated in Fig. 1. For example, living organisms have multiple systems (e.g. reproductive systems), where each of these systems are composed of organs, which in turn are created from tissue, and tissue are created by cells. Each of the components of each layer have a number of different functionalities that enable these components to operate and function independently or cooperatively. However, the hierarchical biological system doesn't just cease at the level of the individual organism. Going up the hierarchy, we will find that different heterogeneous organisms are able to co-exist and live symbiotically. The ultimate inspiration at this scale is available by observing human society. As shown in this hierarchical structure, bio-inspired processes can be categorised into three levels, going from the molecular scale, to the organism scale, with the highest layer being the societal scale. Therefore, using this form of categorisation, we can understand how multiple processes are able to co-exist with minimal conflicts. At the same time, some form of synchronization can also be achieved to allow the different processes to operate in parallel. For example, the bio-inspired processes operating at the molecular scale can occur at frequent intervals, where the end results could be passed to a process operating at the organism level.

In some societies of living organisms, co-existence between the different living organisms are established through some form of mediators. A good example of this is the model used to allow human beings of different societies to co-exist through governments. Governments are usually viewed as central authorities that mediate and negotiate with other governments in order to improve each society's well-being. These 


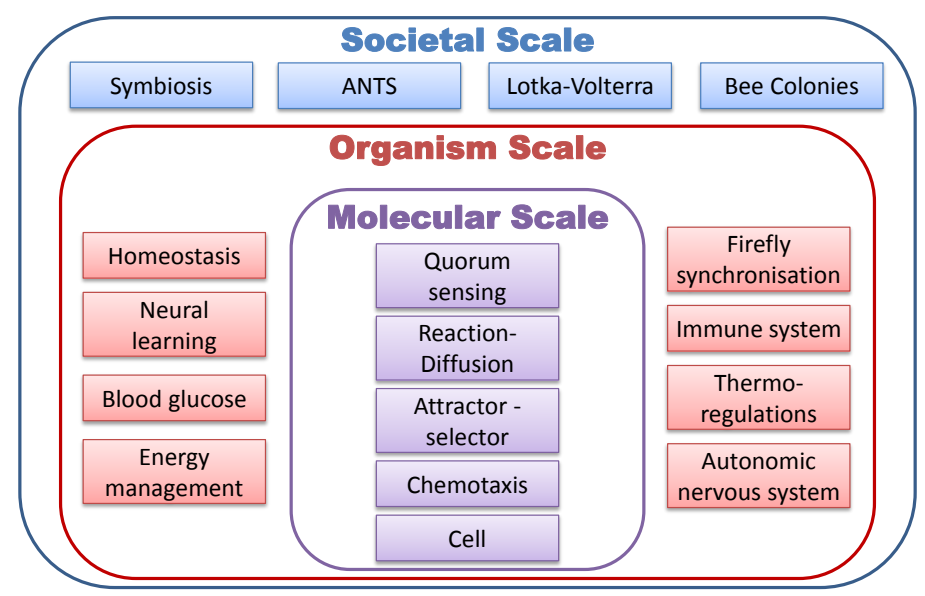

Fig. 1 Hierarchical categorisation of biological processes from the molecular, to organism, to societal scales.

negotiations may include trade negotiations for resources used to support each individual society. As depicted in Fig. 2 this will be the basis of our proposed model for federating systems employing bio-inspired processes.

We envisage an Internet evolution in which flexible and dynamic federation enables cross-organizational inter-operation and cooperation, down to the network level. Within each of the pictured organizations, various adaptable protocols would be deployed, and in our specific case, these adaptable protocols are based on bio-inspired processes. The bio-inspired processes can be deployed in various layers of the protocol stack, with the federation lifecycle management process enabling different entities (in this particular example providers) to share capabilities across organisational boundaries. We see the following as the main challenges that need to be addressed in order to facilitate this form of federation:

- Challenge 1: Integrated Bio-ispired Systems

Applying multiple bio-inspired processes to single/multiple communication systems brings along a new challenge in the design process, as incompatible processes can lead to possible conflicts. Therefore bio-inspired system designers are required to understand biological processes from a systems view, ensuring that different processes are compliant and compatible. The natural hierarchical biological system, as

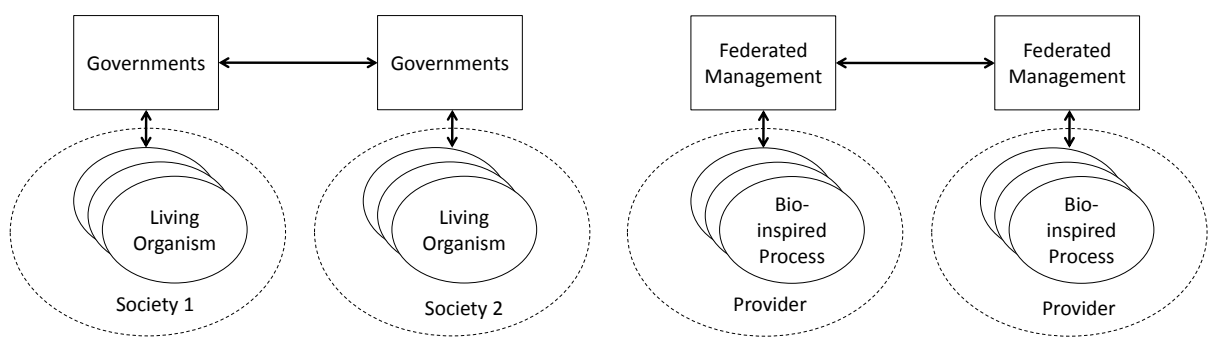

Fig. 2 Analogy between governmental interaction to mediate societies and the proposed federation model for mediation of bio-inspired process in Internet systems. 
exemplified in Fig. 1, can provide a guide for bio-inspired designers when selecting the right process for application;

- Challenge 2: Interface between bio-processes and federated management system Federations enable different parties to use and manage certain resources of other providers: an interface is required to allow federated management systems to interface to biological processes. This brings along a new challenge, since as we add new bio-inspired processes, the federated management system is required to know the new process as well as the parameters that are allowed to be manipulated by the other providers;

- Challenge 3: Federating Management Systems

Federating management systems to allow domain administrators to exercise management functionality on third-party resources is itself a significant challenge. The proliferation of Service Oriented Architectures, web-service APIs on the Internet have made it considerably easier to integrate data from diverse systems into unified cross-domain processes. However, these technologies principally assist syntactic integration - semantic integration remains a significant challenge. What is more, providing sufficiently powerful and fine-grained access control and security management over resources that are partially managed by third parties is generally beyond current management systems. Solutions to this challenge must also be robust enough to deal with high levels of organisational dynamism as real world organisations may change their relationships and the resources that they share with third parties frequently.

These challenges are general in nature, requiring innovative solutions to a number of network and service management problems. The contribution of this paper is to outline a framework and model for management of federations, together with an illustration of how it could be instantiated to provide a content distribution solution for IPTV providers. We thus concentrate on how to federate management systems (challenge 3) and, to a lesser extent, how to have these systems explicitly govern the behaviour of bio-inpired management processes (challenge 2).

\section{Managing Federations}

We view a federation as a cross-organizational agreement in which multiple organization, providers, domains are able to share capabilities in a controlled manner. The objective of such arrangements is to provide the ability for each entity to improve its functionality and capability through such agreements. The end result of this will also be improved end-to-end quality for the end user (eg. a video stream that traverses through multiple networks may end up with degraded quality if one of the intermediate networks is highly congested). Through federation, agreements are arranged between different entities. This agreement enables members of the federation to have certain control of capabilities of other entities within a certain constraint.

In order to realise the vision described above, we have developed a Federal Relationship Management ( $F R M$ ) system. It maintains a set of internal models which capture the dynamic relationships between domains, with a focus on managing secure cross-domain capability sharing and semantic interoperability. At the core of the FRM is a domain relationship model and a capability model. Each FRM manages a set of domains, with each domain being administered independently, and a set of relationships between these domains. Policies can be applied to relationships, which govern 


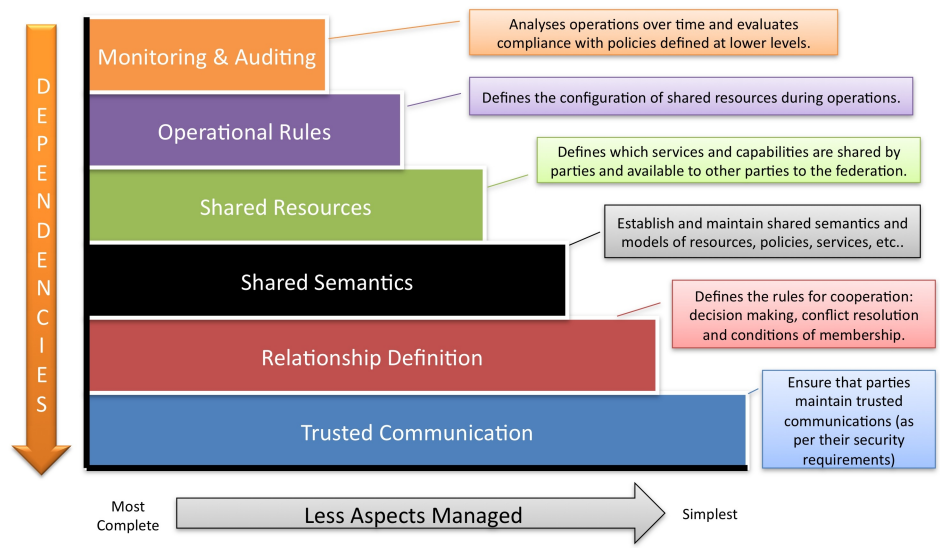

Fig. 3 Layered Federation Model, illustrating what functionalities/models need to be in place in order to support federations of varying scope.

how capabilities can be shared between the related domains. Capabilities are shared between domains by a domain administrator delegating a capability authority to a related domain. These capability authorities are cryptographically signed credentials which contain within them a URL reference to a node on the delegating FRM's capability model. The capability model provides semantic descriptions of capabilities exported by local domains and a data access service which allows those who are delegated capabilities to invoke these services. The inter-operation of the domain relationship and capability models allows domain administrators to exercise fine-grained policy control on resources that are shared with third-parties. A design goal of the FRM was to minimize the requirement for common information models across federations and thus the third and final core model maintained by the FRM is a semantic mapping model. The semantic mapping model describes how imported capabilities are mapped into the local management information model and conversely, how local capabilities are exposed when they are exported.

The FRM software system is an instantiation of the Layered Federation Model (LFM) [21], a conceptual model with each layer representing a specific aspect of an inter-organisational capability sharing agreement, illustrated in Fig. 3. Each layer builds upon and depends upon the lower layers - creating a richer, more robust and more complete description of the aspects of a federal relationship that must be managed in order to enable flexible, dynamic and persistent inter-domain capability sharing relationships. The FRM provides domain administrators with the ability to manage their relationships across these layers. Domains can consume and provide capabilities to and from third-party domains in such a way that minimises the requirement for shared technology and shared models between federating domains, while still being expressive and flexible enough to adequately capture the diverse security and access control requirements of participants. 


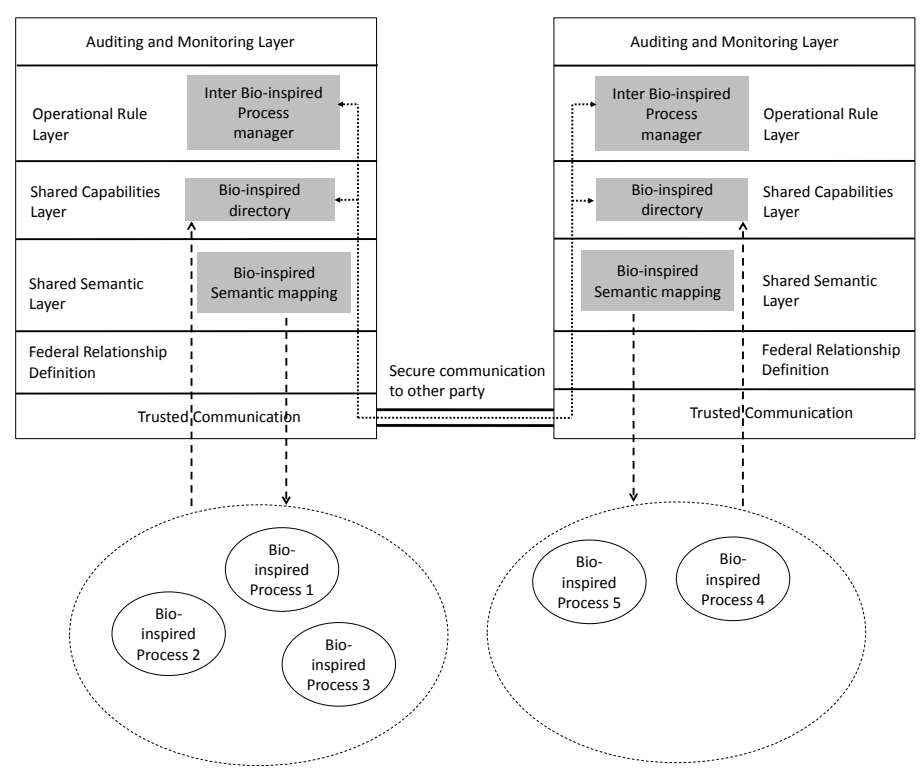

Fig. 4 Extended Layered Federation Model, incorporating cooperative control of bio-inspired networking processes, enabled by bio-inspired directory and semantic mapping processes.

\subsection{Bio-inspired Layered Federation Model}

We have extended the LFM layered model to insert functionalities to support the bioinspired processes in the underlying networks; this is illustrated in Fig. 4. The shared semantic layer enables mappings between the semantic representation of capabilities and resources that each organization possesses. Therefore, within this layer, the Bioinspired semantic mapping process enables this mapping process to occur between different organizations. Since each organization will apply various bio-inspired processes to manage and control their own resources, the Shared Capabilities layer includes a Bio-inspired directory that describes the various processes that are deployed in each organization's networks. The Inter Bio-inspired Process Manager, situated in the Operational Rule Layer, allows each organization to influence through parameterization the bio-inspired processes of other federation members.

\section{Case Study: IPTV Content Distribution}

Our case study is illustrated in Fig. 5. In this scenario there are six different organizations that collaborate to provide an IPTV service to subscribers in Ireland (federation membership is depicted in Fig. 6). One of the organisations, the Cork storage provider, is initially not involved in service delivery but is dynamically added (as described below) in response to subscriber demands and network load conditions. There are two federations used to deliver the service: an overall IPTV service federation which is controlled by the IPTV service provider and a storage provider federation that consists of just the Dublin, Waterford and Limerick storage providers who have a business agreement to increase the efficiency of their operations by sharing server load and availability 


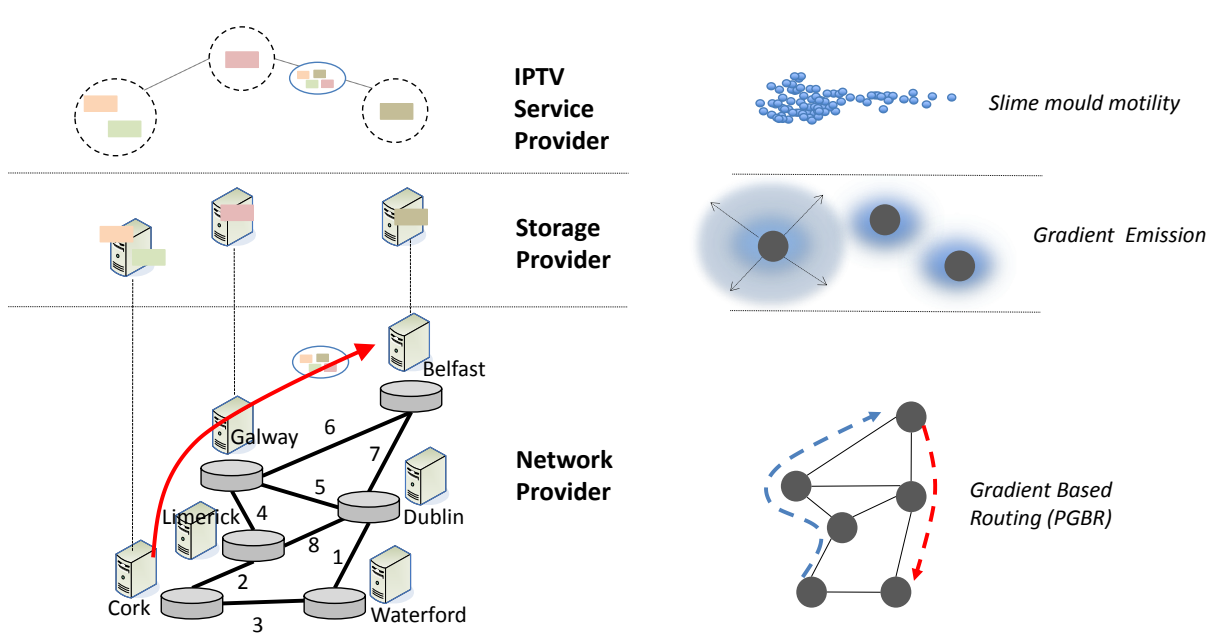

Fig. 5 Case study depiction, showing the network topology on the left and the deployed bio-inspired processing types on the right.

advertisements via a common bio-inspired gradient-based process. The IPTV service provider is responsible for subscriber and session management of IPTV sessions but has no network or content storage resources of its own, these are leased from a network provider and regional storage providers on an as needed basis. To illustrate our contributions in a reasonable space, we take a slightly simplified case where all session control is centralised in the IPTV service provider domain, of course this session control could also be provided by decentralised capabilities that are leased by each storage provider or another third party.

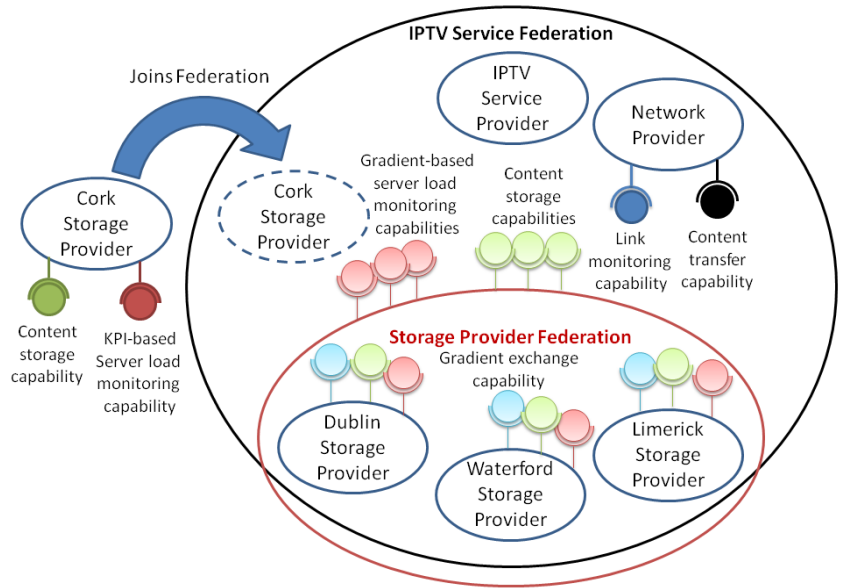

Fig. 6 A capability sharing and federation membership view of the case study; a new storage provider based in Cork is added to the federation in order to meet additional demand for IPTV content streaming. 
Within each federation, providers can share capabilities, such as access to monitoring information or content management or transfer services. As described earlier, the autonomic management processes to date have largely focused on developing selfmanagement mechanisms for individual networks or sub-systems. In this case study, we will show how diverse bio-inspired mechanisms can co-operate across multiple organisations, while using the FRM as a mediator to provide interoperability and consistent enforcement of business level agreements right down to the network level. In the following description we ignore accounting or billing related capabilities that are also shared between domains, but they can be shared using the same FRM mechanisms that we outline here.

There are three bio-inspired processes in the case study, as illustrated in Fig. 5. In the Network Provider, a Parameterised Gradient Based Routing (PGBR) process is used to stream content traffic through the network. This PGBR routing algorithm is a gradient based routing process, where routes are discovered hop-by-hop, and is inspired by the chemotaxis process (chemotaxis is a bacteria motility process, where bacteria migrate following a chemical gradient). Full details of the PGBR routing algorithm can be found in [6]. At the IPTV service federation level this process is transparent to its consumers but business-agreement based access controls are applied to it through the sharing of a content transfer capability. In addition the Network Provider shares a link monitoring capability so that the consumers of this service can monitor network behavior and calculate customer QoS/QoE metrics.

The second bio-inspired process is deployed within the storage provider federation, where each member Storage Provider uses a gradient emission process, to estimate the load on its servers and to advertise their availability for customers. To enable inter-provider advertisements each storage provider in the federation shares a gradient exchange capability. As a whole, the storage provider federation shares two types of capabilities with the IPTV service federation: content storage capabilities that allow consumers to store and manage content on the storage providers servers and server load monitoring capabilities that are based on the results on the actions of the gradient emission process.

The IPTV service provider uses a slime mould bio-inspired process for content replication management, to optimise the location of content with respect to service demands, storage provider availability and network link load. This process enables popular content to migrate as a group from storage provider to storage provider. Thus the slime mould content replication process needs to consume the Network Provider link monitoring and Storage Provider server monitoring capabilities as inputs to its decision process. However rather than tightly coupling a particular metric publication scheme to the decision process, the semantic capability descriptions published by the Network and Storage provider FRMs are used to enable semantic mapping between these external models and the IPTV service providers internal models for server and network load/availability and cost, which the slime mould process can manipulate directly. This isolates the model dependence of the bio-inspired autonomic control and reduces the integration effort required to consume new provider metrics. This also lowers the bar for standardisation, since if the IPTV content provider only uses storage provider capabilities on a dynamic basis it is only necessary to integrate with them on an as-needed basis i.e. there is no need for overarching protocol syntax and semantics agreements with third parties with which the IPTV service provider does not wish to do business. Similarly, in order to execute content re-distribution actions, the IPTV service providers slime mould replication process must consume the content 


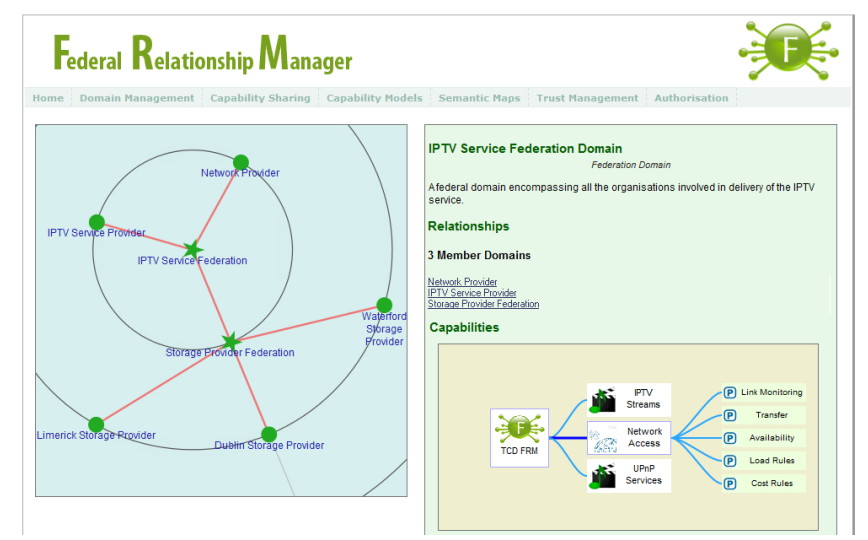

Fig. 7 A screen snapshot of the Federal Relationship Manager interface; a visualisation of federation membership is shown on the left, whilst the right shows details of capability models.

management and transfer capabilities provided by the storage providers and network provider respectively. These are also mediated through the FRM capability models and could take different forms for different providers, e.g. FTP-based content transfer or web service-based content transfer. Fig. 7 shows a screenshot of the FRM, containing a snapshot of both the domain relationship model and the capability model.

The scenario under investigation in this case study is one where the IPTV service provider initially has their content distributed across the servers of the storage provider federation. The content in Dublin is in demand from subscribers in Waterford and hence is loading the Network Providers link 1 in Fig. 5. The IPTV service provider then determines that this demand is going to continue to grow and that the Limerick storage provider has limited or no availability for additional content. Hence it decides to form a new business agreement with the Cork storage provider and migrate content there to ensure that it can continue to satisfy the increasing demand from Waterford. Since the Cork storage provider is not a member of the storage provider federation or the IPTV service federation it must be invited to join the IPTV service federation. The Cork storage provider does not implement the gradient emission process to share server availability advertisements and hence it has no reason to join the storage provider federation. However it does have a more traditional KPI-based availability measure that it exposes through a shared capability and some content storage capability that it also can share. Through a semantic mapping process at the FRM the IPTV service provider can ensure that its slime mould content replication process can consume these capabilities in a way that is equivalent to the capabilities shared by the members of the storage provider federation. Hence the slime mould content replication process can transparently use these capabilities to transfer content across the different storage providers. Once the demand surge from Waterford is dealt with, the Cork storage provider can be removed from the IPTV service federation.

The sequence diagram of interactions between the key providers for our use case is shown in Fig. 8. The stable interactions with members of the storage provider federation are not shown and after the first capability use (a request for the Cork storage providers server load monitoring information by the IPTV service provider) we simplify the capability use and access control requests since they all follow the pattern illustrated for the first request. 


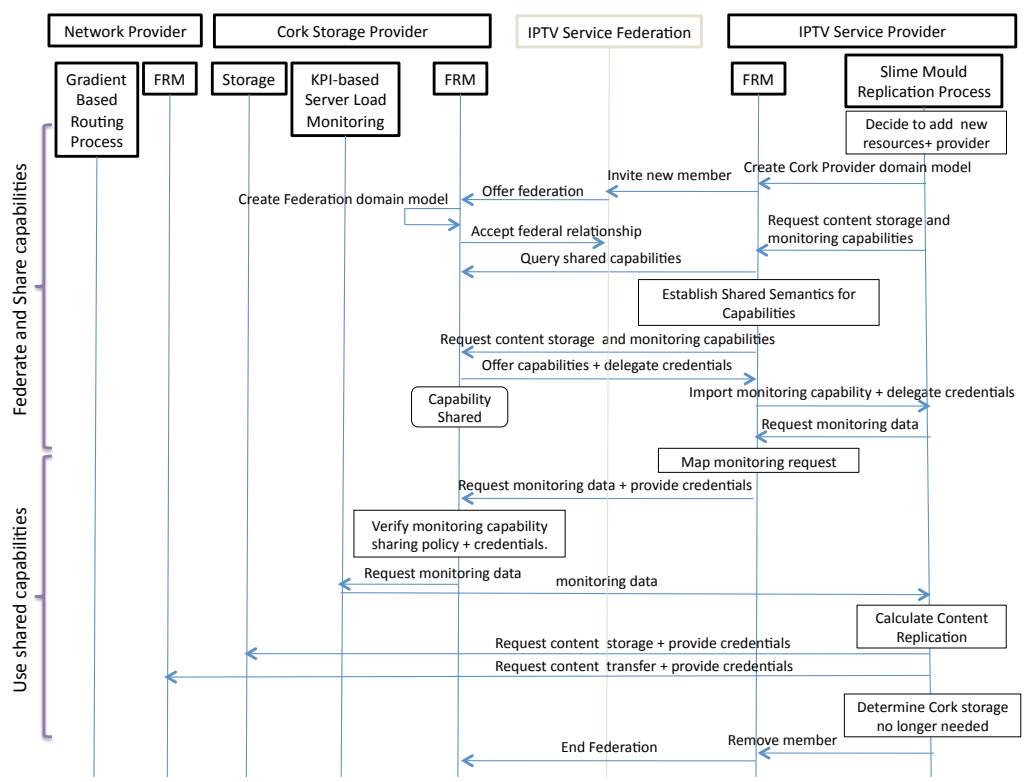

Fig. 8 Federation formation and capability use message sequence chart.

The figure is divided into two phases (indicated on the left hand side of the diagram): Federation of the Cork providers monitoring and data storage capabilities and then use of the shared capabilities. In the first phase the IPTV service providers slime mould replication process manager, or a higher level manager working on its behalf, creates a FRM request to add the Cork storage provider to the IPTV service federation, creating a model of that domain in the FRM and setting any applicable local policies. Since the IPTV service provider is the controller of this federation it has the authority to invite new members and this invitation is passed on to the FRM of the Cork storage provider. This in turn requires a domain model and local policies to be created in the Cork FRM and an accept notification is sent. At this point the Cork storage provider is a member of the federation but no capabilities have been shared. The IPTV service providers slime mould content manager then requests the monitoring and storage capabilities it requires. This is then sent to the Cork storage providers FRM in the form of a shared capability query to determine if appropriate capabilities are available. The query results are subject to semantic analysis in the FRM to determine whether any of the available capabilities can be matched to the desired capabilities and what, if any, import semantic mappings must be deployed in the IPTV service provider to use them. Given that federation agreements may have existed before between these domains, it is likely that mapping discovery based on the capability description and federation context will suffice to locate appropriate mappings from the FRM mapping repository. Otherwise a semantic mapping process will have to be initiated, that may require human intervention. Once a shared semantics for the capabilities have been established the IPTV service provider can request the specific capabilities desired. Finally the storage providers capability sharing offer and delegation authority are encoded as 
X.509/Keynote credentials and sent to the IPTV service providers FRM. At this point the IPTV service providers FRM can make the monitoring capability available to the slime mould content replication process.

Then we move to phase 2 of the figure, capability use. Now the content migration process can request monitoring information from the Cork storage providers FRM since it has appropriate credentials. Once the request arrives at the storage providers FRM it checks the credentials are valid (against its access control policy and any other local restrictions i.e. sharing policies or operational rules). Then if export mappings were applied by the Cork FRM when it originally shared the capabilities, it reverses them to map the request into the local management model and sends the request to the local storage manager. This may result in further local interactions that are not illustrated within the storage provider domain and then the monitoring data is returned to the IPTV providers content migration process. Once the content migration process wishes to move content onto the storage provider a similar process is followed and the data is migrated to the Cork storage providers servers. This also requires a request to the Network Provider to transfer this content. In reality this network provider capability shared with the IPTV service provider may be delegated from the IPTV service provider to the storage provider within a FRM capability delegation chain but the simplier case of direct control by the IPTV service provider is shown here for clarity.

\section{Illustration of Slime Mould Content Replication}

In this section we illustrates the benefits of using the slime mould content replication process to replicate content across multiple storage servers. For example in cases where particular content items are very popular (for example following the release of a blockbuster movie) the increasing volume of requests will be detected and content replicated to multiple storage servers from where it can be streamed under the control of a server selection / admission control process. Alternatively, in cases where the network links connecting users to the IPTV service provider's "home" server become congested, content can be replicated to other servers to ensure that users' requests can be admitted and receive adequate quality-of service. If we assume that storage providers charge for use of their servers on a storage capacity used per unit time basis then there is a clear cost saving if content is only replicated by the IPTV service provider in circumstances where otherwise users' requests would be rejected.

Our slime mould inspired content replication process takes into account the volume of requests/rejections for particular content item classes as well as end-to-end available bandwidth estimates provided by storage providers. Cellular slime moulds [1] are single cell amoebiod organisms that have the ability to aggregate together upon release of a chemical signal in order to form an aggregate that can move as if it were a larger organism. This behaviour has been harnessed to solve a range of optimisation problems, for example planning an urban rail network [36]. In our analogy individual IPTV content items are the single cell slime moulds, which upon the release of a signal (higher request rejection rates) aggregate together and replicate themselves on a remote storage server.

Fig. 9 shows a simplified version of the case study scenario described in $\S 4$. We assume that a federation is instantiated by two FRMs between an IPTV service provider that has its own storage server and a server selection / admission control server and 


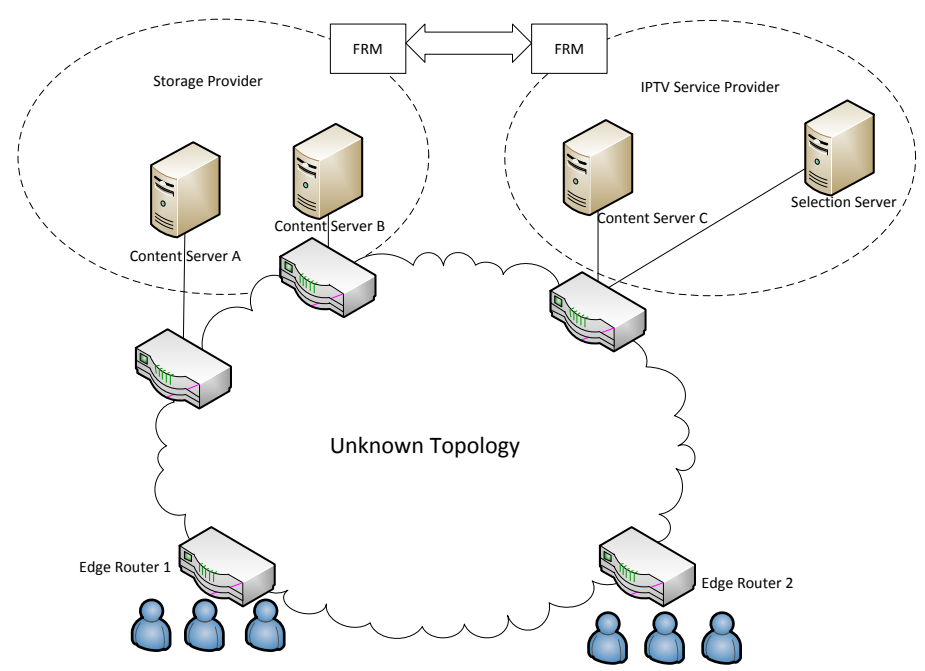

Fig. 9 Simplified IPTV Content Distribution Federation Scenario; the Selection Server performs admission control, server selection and slime mould based content replication based on analysis of content item request rejection rates and available bandwidth estimates from content servers.

a Storage Provide offering storage in data centres with two separate network points of attachment. The storage servers have monitoring capability in the form of available bandwidth estimation tools that can be configured to use packet probes to estimate the available bandwidth between their network point of attachment and the network points of attachment of various user groups. We assume that the network provider is not part of the federation, so the IPTV service provider and storage provider do not have knowledge of the network topology or access to more detailed network monitoring data. As shown in previous work $[27,28]$ available bandwidth estimations are a suitable means of monitoring quality of service and controlling end to end delay for the purposes of IPTV admission control.

Users IPTV clients request streaming of content items from the selection server. The selection server decides to admit the request if at least one content server that currently contains the item estimates that there is enough bandwidth between it and the user's point of attachment to serve the request. If more than one content server is capable, the server with the most available bandwidth is chosen. Rejections occur when there is not enough bandwidth available on any path to ensure servicing the request with adequate quality-of-service.

The ability to replicate and remove content as required can significantly aid in increasing requests accepted. It also has the advantage of improving costs by ensuring efficient use of resources. With the slime mould inspired replication algorithm Iitems are chosen for replication based on the amount of rejections they have experienced in the preceding control interval. If the amount of rejections for an item (as a percentage of the overall requests) is greater than a set threshold, then that item is marked for replication to another content server if a server is available in the system to host it. Similarly, if an item has experienced an amount of rejections in the last time interval that is less than a pre-determined minimum amount, then it is removed from one of the content servers hosting it; this prevents the situation where revenue is spent on 
Table 2 Notation for Slime Mould Inspired Content Replication Algorithm

\begin{tabular}{cl}
\hline Notation & Description \\
\hline$i$ & A particular content item group \\
& The number of content item groups offered by the service \\
$s_{i}$ & provider \\
$\left(t-t^{\prime}, t\right)$ & The storage capacity required for content item group $i$ \\
$q_{t o t}\left(t-t^{\prime}, t\right)$ & The time interval for which requests and rejections are recorded \\
& $\left.t^{\prime}, t\right)$ \\
$r_{i}\left(t-t^{\prime}, t\right)$ & Number of requests for content item group $i$ that rejected during \\
& $\left(t-t^{\prime}, t\right)$ \\
$R F_{i}$ & The current rejection rate of content item group $i$ \\
$J$ & The number of available content servers \\
$j$ & A particular content server \\
$S_{j}$ & Storage capacity currently available on content server $j$ \\
$d$ & A particular edge router \\
$D$ & The number of edge routers \\
$\hat{A}_{j d}$ & The available bandwidth estimate between content server $j$ and \\
$\left\{i_{j}\right\}$ & edge router $d$ \\
$n(i)$ & The set of content item groups stored on content server $j$ \\
$R F_{\min }$ & The number of servers currently storing content item group $i$ \\
$R F_{\max }$ & Minimum threshold for acceptable rate of rejections \\
& Maximum threshold for acceptable rate of rejections \\
\hline
\end{tabular}

operating servers that are under utilised. The slime mould inspired content replication algorithm is specified in Alg. 1 with the notation used described in Table 2. Each time it executes the algorithm loops through the content item groups and for each content item group loops through the number of content servers two times. Therefore it is $\mathcal{O}\left(\backslash^{\epsilon}\right)$. Since we propose a possibly large number of content items are grouped in to a relatively small number of content item groups we believe the time complexity of the algorithm would not be a significant issue.

\subsection{Simulation Study}

We now present the results of a simulation study of the operation of the slime mould inspired content replication process. The modelled network topology used is depicted in Fig. 10. It consists of three content servers and a selection server. Background loads have been added to the paths to create the steady state mean available bandwidth profiles shown in Table 4 . The base content server in the topology is server $\mathrm{C}$ and there are two other content servers (servers A and B) to which content items can be replicated to under the control of the slime mould inspired content replication algorithm. Requests come into the network from two points of attachment (Edge Routers 1 and 2). The items in the IPTV content library have an average length of 20 mins. Replications are triggered every 600 seconds. We group individual content items into 15 content classes (treated as single units for replication purposes) and model request arrivals at different rates for groupings of 5 content item classes. Overall, requests occur in steady state at an expontentially distributed rate of $0.08 / \mathrm{s}$. To examine the performance of 


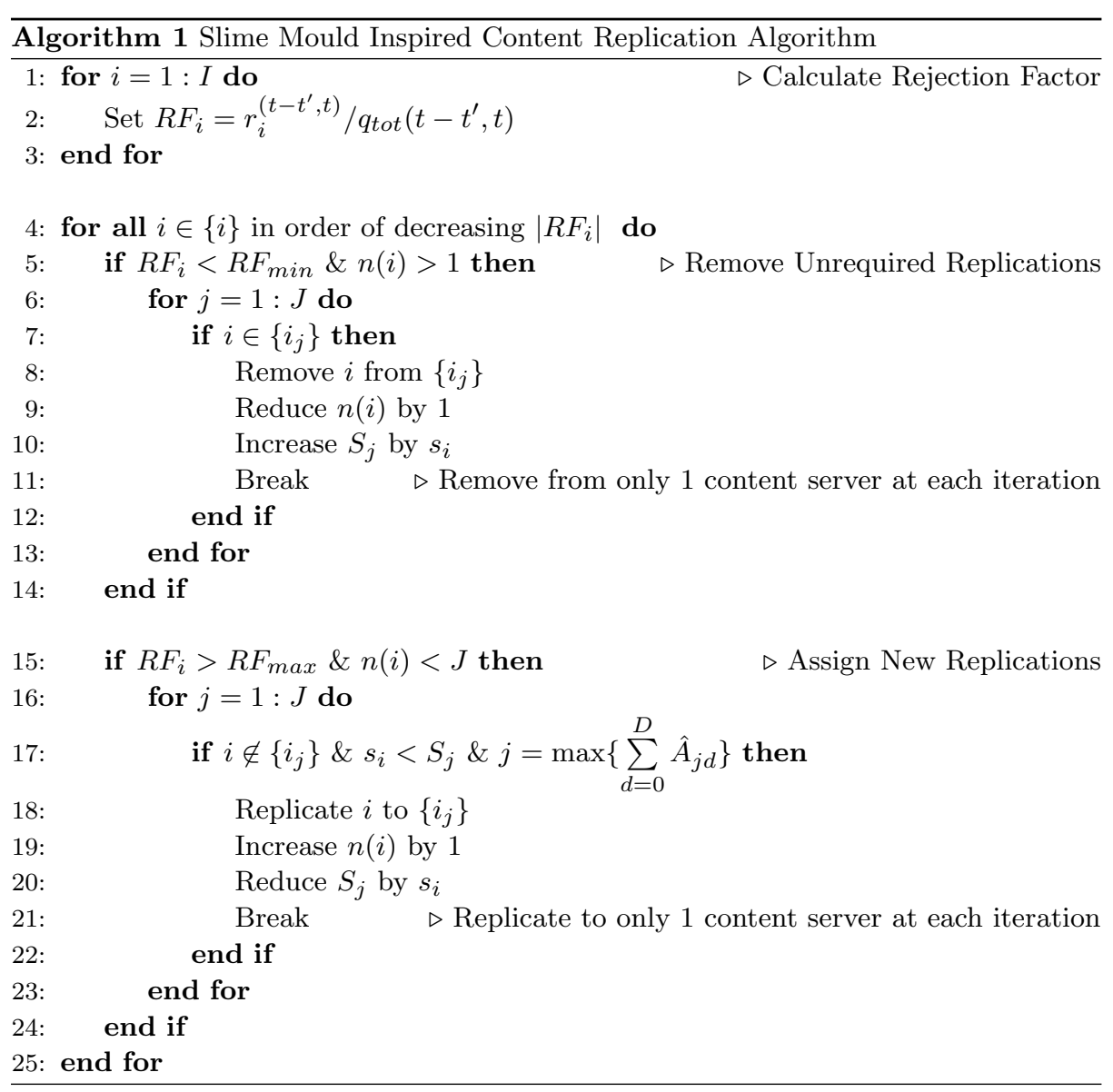

the replication algorithm, two scenarios were developed: where there was a significant increase in the rate of requests, and one where there was a significant degradation in network conditions. To further illustrate the benefit of using this bio-inspired content replication process, a comparison is undertaken in each of the scenarios with an approach that uses a non bio-inspired replication process, formally specified in Algorithm 2 and Table 3. This comparison algorithm operates by monitoring the rejections in the system and when a threshold is reached, randomly selecting a subset of content items classes to replicate.

\subsubsection{Increased Request Rate Scenario}

To test the replication algorithm, a step change in the rate of requests was introduced. Starting at 7200 seconds, the request rate of one of the three sub groups was increased to 0.35 requests per second, with exponentially distributed inter-arrival times. Fig. 11 shows the rate of accepted requests for the situations where bio-inspired and non bioinspired replication occurs as well as where replication not occur. It can be seen that the grouping of content item classes by the slime mould based replication allows more 
Table 3 Notation for Randomly Selected Content Replication Algorithm

\begin{tabular}{|c|c|}
\hline Notation & Description \\
\hline$i$ & A particular content item group \\
\hline$I$ & $\begin{array}{l}\text { The number of content item groups offered by } \\
\text { the service provider }\end{array}$ \\
\hline$\left(t-t^{\prime}, t\right)$ & $\begin{array}{l}\text { The time interval for which requests and re- } \\
\text { jections are recorded }\end{array}$ \\
\hline$q_{t o t}\left(t-t^{\prime}, t\right)$ & $\begin{array}{l}\text { Total number of requests for content items ar- } \\
\text { rived during }\left(t-t^{\prime}, t\right)\end{array}$ \\
\hline$r_{i}\left(t-t^{\prime}, t\right)$ & $\begin{array}{l}\text { Number of requests for content item group } i \\
\text { that rejected during }\left(t-t^{\prime}, t\right)\end{array}$ \\
\hline$r_{t o t}\left(t-t^{\prime}, t\right)$ & $\begin{array}{l}\text { Total Number of requests that were rejected } \\
\text { during }\left(t-t^{\prime}, t\right)\end{array}$ \\
\hline$J$ & The number of available content servers \\
\hline$j$ & A particular content server \\
\hline$S_{j}$ & Storage capacity available on content server $j$ \\
\hline$s_{i}$ & $\begin{array}{l}\text { The storage capacity required for content item } \\
\text { group } i\end{array}$ \\
\hline$\left\{i_{j}\right\}$ & $\begin{array}{l}\text { The set of content item groups stored on con- } \\
\text { tent server } j\end{array}$ \\
\hline$n(i)$ & $\begin{array}{l}\text { The number of servers currently storing con- } \\
\text { tent item group } i\end{array}$ \\
\hline$i^{*}$ & $\begin{array}{l}\text { List of content item classes that can be repli- } \\
\text { cated }\end{array}$ \\
\hline$R_{\min }$ & $\begin{array}{l}\text { Minimum threshold for acceptable rate of re- } \\
\text { jections }\end{array}$ \\
\hline$R_{\max }$ & $\begin{array}{l}\text { Maximum threshold for acceptable rate of re- } \\
\text { jections }\end{array}$ \\
\hline
\end{tabular}

requests to be accepted as it prioritizes popular content items for replication. Fig. 12 shows the bandwidth available from each server to one of the points of attachment. Initially requests are allocated to the base server. Replication of the highly requested item classes occurs to this server and admission control and server selection is performed based on the path with the most available bandwidth. As the higher request rate is maintained, the storage provider informs the IPTV service provider that the paths from both the base server and server B have become degraded and another server, server $\mathrm{C}$ is used for content replication. When the step change in the rate of requests is completed,

Table 4 Link Available Bandwidths.

\begin{tabular}{ccc}
\hline Server & Edge Router & Available Bandwidth (Mbps) \\
\hline A & 1 & 50 \\
B & 1 & 40 \\
C & 1 & 70 \\
\hline A & 2 & 50 \\
B & 2 & 40 \\
C & 2 & 70 \\
\hline
\end{tabular}




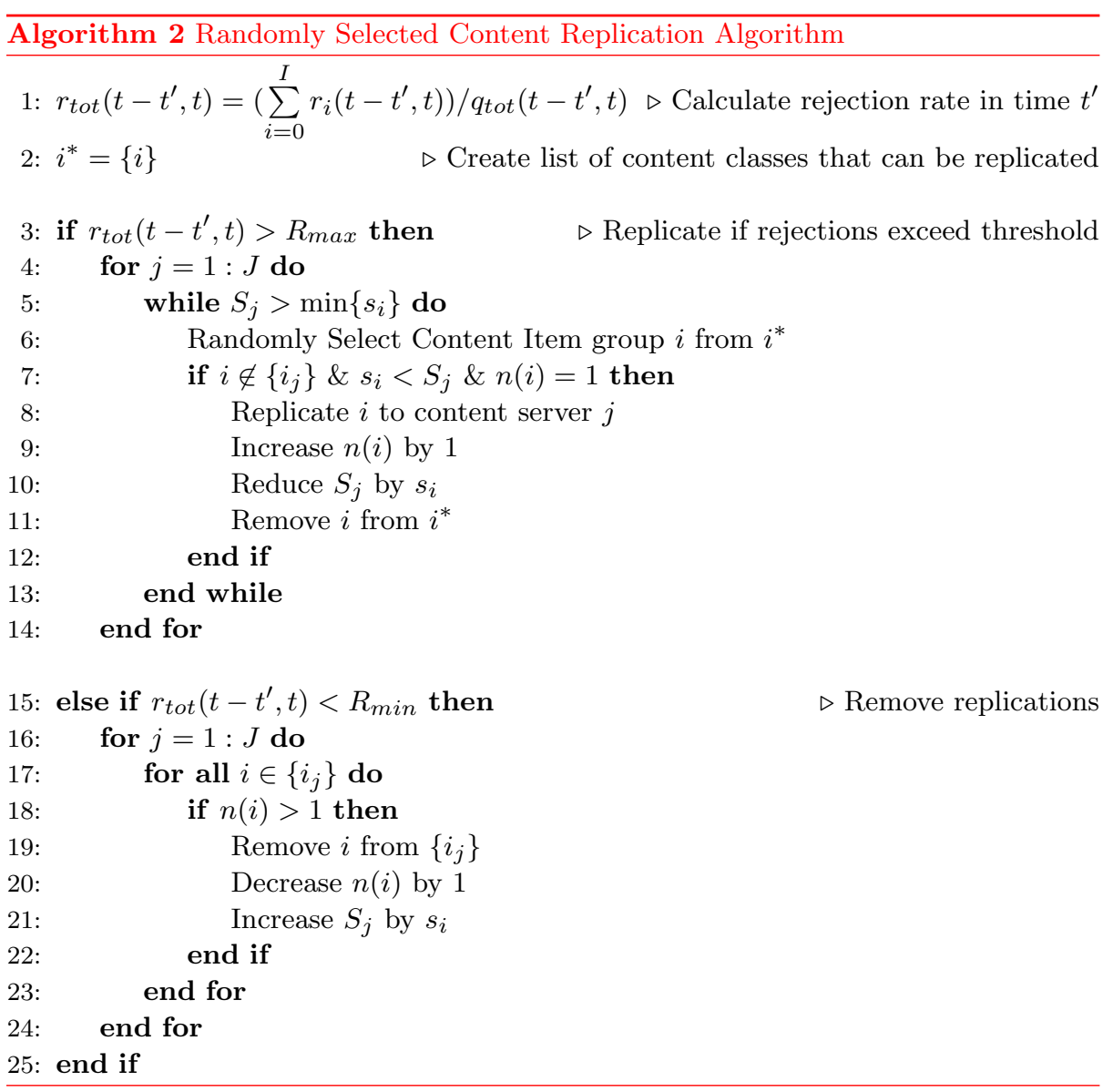

the decrease in request rejections is detected and content is removed from the servers A and B, thus prevent unnecessary utilisation of resources (and associated cost), as shown in Fig. 13(b). It is apparent that intelligently replicating content in times of high utilization allows the IPTV service provider accept more requests overall, while maintaining the quality of the accepted requests. It can also be seen that this approach helps in the efficient use of resources as only currently popular content is replicated, thus any resources rented on additional servers are actively being used and providing value.

\subsubsection{Increased Background Traffic Scenario}

If there is a increase in the background traffic load in the network, fewer requests can be admitted by the selection server if users' quality-of-service is to be maintained. In this case the ability to replicate content to other less congested locations can help maintain the revenue generated by accepting requests. To examine the performance of the replication approaches in such a scenario, the rate of requests was kept at its steady state throughout but a step increase in the background traffic on the network paths 


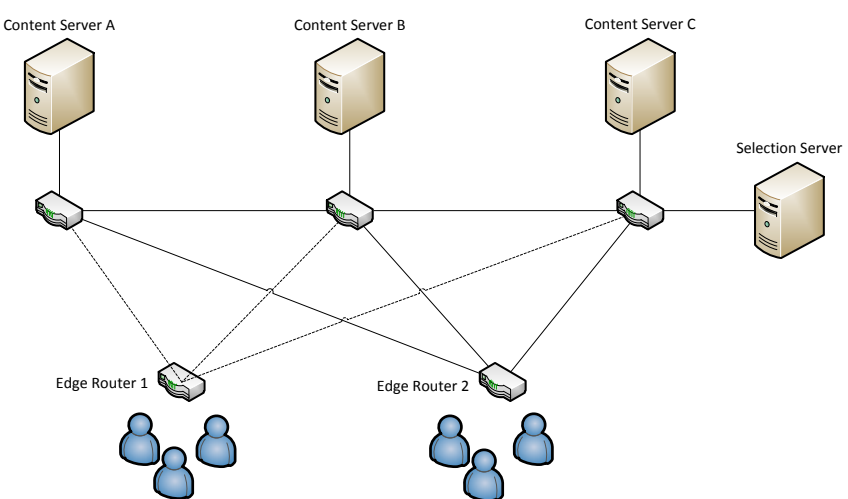

Fig. 10 Simulated Network Topology.

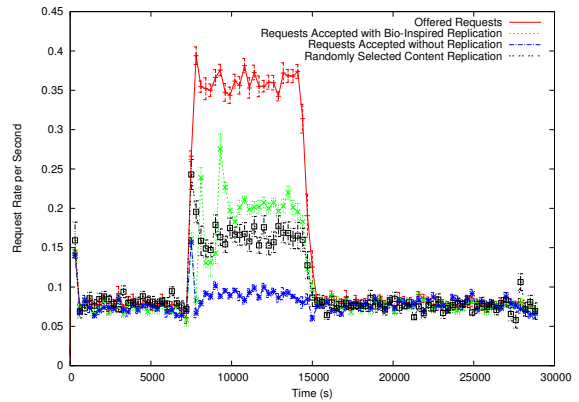

Fig. 11 Requests Accepted during a step increase in request arrival rates for one of the three groups of content item classes. Because the slime mould replication algorithm replicates popular content items it accepts more requests during the overload period. Results presented are averaged from 10 independent simulation runs.

between the edge routers and two of the three storage servers was modelled. Table 5 shows the changed available bandwidths that are available during this time period.

Fig. 14 shows the requests accepted for this scenario. When the background traffic increases, the ability to replicate allows the system to accept requests at a rate which is more beneficial than when replication does not occur. Fig. 15 shows the bandwidth estimates reported for the three servers to edge router 1 . When the step change occurs initially, the base servers capacity to accept new requests is negligible. This leads to item classes requiring replication even though their popularity has not changed noticeably. The replication occurs to one of the sub servers which are brought into the federation by the FRM and the capacity on the paths from this server are then allocated. As rejections in the system are still occuring, and the network provider is reporting a very low capacity on existing resources, another server is brought into the federation and the content classes that are experiencing rejections are replicated to here also. At the end of the step change, the network provider reports the improvement in conditions up to the IPTV service provider, and the extra servers are noted to be no longer required. Therefore, the IPTV service provider uses the FRM removes them from operation and can service requests from its base server again. It can be seen that the random replication admits a similar amount of requests to the slime mould based 


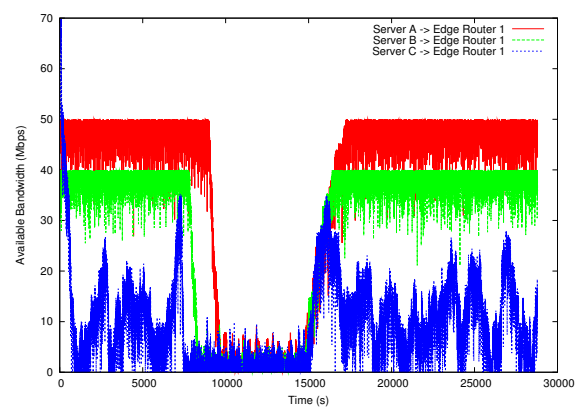

Fig. 12 Bandwidth utilisation for slime mould based replication during a step increase in request arrival rates for one of the three groups of content tem classes for a single simulation run. As content items are replicated the algorithm accepts only the number of requets that can be satisfied given the available bandwidth to the three servers.

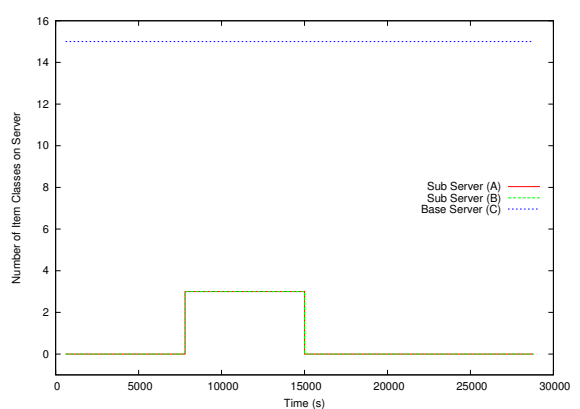

(a) Random Replication

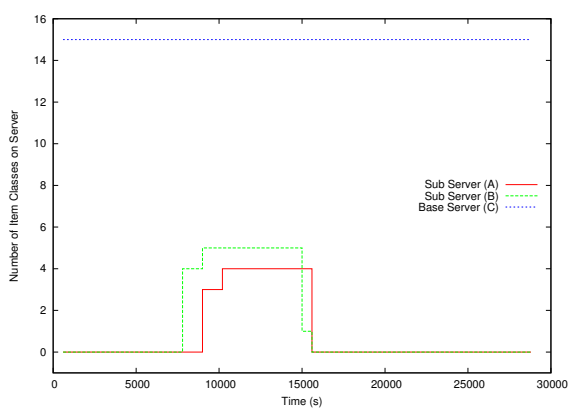

(b) Slime Mould based Replication.

Fig. 13 Server content item class occupancy during a step increase in request arrival rates for one of the three groups of content tem classes for a single simulation run. for the Random algorithm a randomly selected set of content items is replicated to each of the servers when the overload is detected, and removed once it abates. For the slime mould based content replication algorithm the content item classes with high request rates are first replicated to server $\mathrm{B}$, then to server A, and removed once the step increase abates.

replication. This is due to the fact that none of the content item classes are experiencing a noticeably higher request rate than any other, and therefore the strength of grouping and replicating high frequency items is negated. The slime mould replication algorithm is still more beneficial to the IPTV provider though, as can be evidenced in the content item occupancy shown in Fig. 16.

\section{Related Work}

A number of bio-inspired approaches have been applied to communication network and services [29]. Suzuki and Suda [35], developed a bio-inspired autonomous service solution, where each service unit was modeled to fit an organisms lifecycle (e.g. services had internal energy management, ability to replicate, migrate, and reproduce with other services to produce new offspring). Each service unit was able to house various types of services including web services, video content, etc. By integrating bio-inspired mecha- 


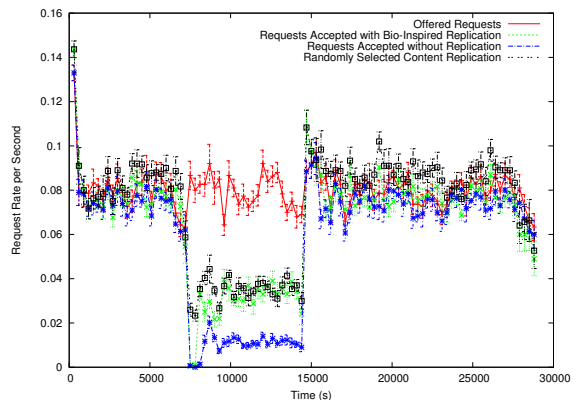

Fig. 14 Requests accepted during increased background traffic on paths to two of the three content servers. Results presented are averaged from 10 independent simulation runs.

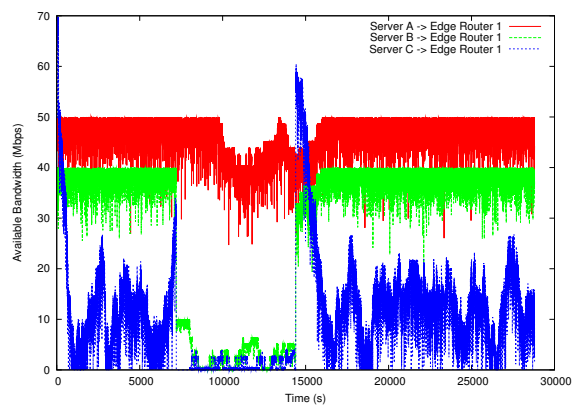

Fig. 15 Bandwidth utilisation for slime mould based replication during increased background traffic on paths to two of the three content servers. Reduced bandwidth leads to increasing rejcetion rates, which triggers replication of content items. Requests are then only accepted for content items hosted at the server to which there is sufficient available bandwidth.

Table 5 Link Available Bandwidths for Increased Background Traffic Scenario.

\begin{tabular}{ccc}
\hline Server & Edge Router & Available Bandwidth (Mbps) \\
\hline A & 1 & 50 \\
B & 1 & 10 \\
C & 1 & 10 \\
\hline A & 2 & 50 \\
B & 2 & 10 \\
C & 2 & 10 \\
\hline
\end{tabular}

nisms, the services were able to autonomously evolve with respect to user demand, as well as network conditions.

In the underlying network, a number of different bio-inspired approaches have been developed to support routing. A very good example is the application of insect colonies, such as ants, where Di Caro and Dorigo [11] developed AntNet which is a protocol developed to discover paths in networks. Besides the use of insect colonies, algorithms inspired from evolution have also been developed for routing. Ahn and Ramakrishna [4] developed a solution that uses genetic algorithms to determine routing paths. Leibnitz et al. [25] developed a solution for self-management of overlay paths, whereby paths would be able to switch from primary to secondary paths when environmental condition 


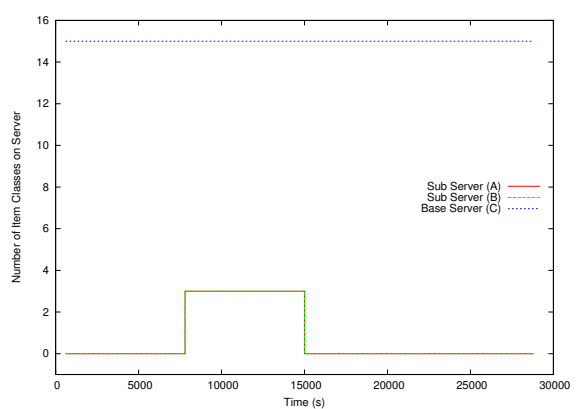

(a) Random Replication.

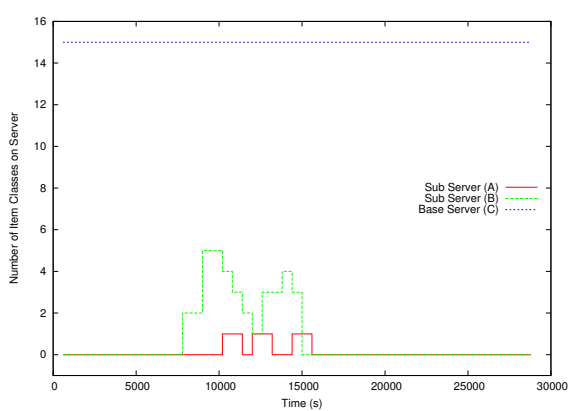

(b) Server Loads.

Fig. 16 Server content item class occupancy during a step increase in background traffic on network paths to two of the three servers. Both algorithms trigger the replication of content items, however the slime mould replication algorithm favours replication to server B as there is limited bandwidth available to serve requests from server A.

worsens (e.g. end-to-end delay). The bio-inspired process used was known as AttractorSelector and is based on the self-management properties found in E. Coli bacteria.

Besides using biological systems to develop algorithms and protocols that could be used for developing autonomous and adaptive communication networks, a new area called biologically driven networks [35] is emerging, where real biological systems can be used to design communication systems. For example Tero et al. [36] used slime moulds to emulate the design of the shortest path topology of the Greater Tokyo railway network.

The bio-inspired process illustrated used a slime mould behaviour inspired algorithm that used monitoring information from end-to-end available bandwidth estimation tools. A network path's available bandwidth has been defined as "its remaining capacity, that is, the amount of traffic that can be sent along the path without congesting it" [10]. Many tools exist for estimating available bandwidth in an end-to-end context; that is, without requiring directly gathering information from the intermediate topology. ABETs such as Pathload [20], Spruce [34], Assolo [17], Yaz [33], and BART [14] all propose different approaches for available bandwidth estimation. In our experiments we have used the PathChirp [32] ABET, which has performed well in such comparisons, has a publicly available implementation and, as shown in our previous work [27], is suitable, when appropriately parameterized, for use in IPTV admission control schemes.

The illustrated bio-inspired process was the foundation for the admission control system used in the IPTV Content Distribution case study. Recently, Admission Control in content distribution networks that requires network level interaction has been published. Latré et al [24] presented an approach to measurement based Admission Control that utilized PCN [2] to gather measurements while Asghar et al [5] presented an Admission Control architecture that used RSVP [3].

From the federation management perspective the proliferation of applications and processes that span organisations has made the challenges of effectively managing them increasingly apparent. Much of the research on cross-organisational management has focused on the specification of contracts and agreements between organisations, which then must be monitored and enforced by both parties, in particular focusing on service 
levels agreements (SLAs) $[8,18,22,30]$. SLA-driven, cross-organization management is typically divided into two phases - SLA negotiation and specification, and then the runtime monitoring SLA fulfillment and delivery, with associated management actions to ensure that the service is being delivered appropriately. Traditionally, defining an SLA is a slow and tedious process requiring complex legal agreement and protracted negotiation. Approaches at defining SLAs in machine interpretable formats have enabled the dynamic interpretation and evaluation of SLAs (see [30] for a comparison of formats). However there has been little progress in the field of automated or dynamic negotiation of SLAs outside of the field of cloud or grid computing [19,39].

Beyond SLA management, researchers are addressing the challenges of developing languages and frameworks addressing cross-organisational (access control) policy management. For example, Organisational Based Access Control (OrBAC) [15] is an access control policy model that is explicitly designed to allow management policies to be applied in a multi-organisational setting. X-FEDERATE [7] goes one step further in that it incorporates a policy language, a UML-based meta-model and enforcement architecture, and a common policy authoring process designed to help administrators from related organisations to arrive at mutually interoperable management system configurations. Whilst these and similar works are promising they are limited to access control, and do not address wider federation challenge, including, as outlined in this paper the use of federation techniques to integrate or coordinate the behaviour of network-level self-management processes.

\section{Conclusions}

The Internet continues to influence our lives and has now become a critical infrastructure that is essential towards meeting a number of our needs. However, with increased management complexities resulting from integration of heterogeneous networks, as well as heterogeneous management domains, the research community is currently seeking new management solutions. In particular, the evolving Internet requires a network and service infrastructure that is highly dynamic, robust, adaptive, and efficient. While the research community have addressed these requirements through autonomic communications, in particular bio-inspired processes, the current solutions are only limited to addressing single communications functionalities that are managed in a single domain. Our main contention is not that bio-inspired processes, either operating individually or in a coordinated manner, are inherently superior to traditional distributed resource allocation approaches (although researchers have shown this to be the case for particular examples). Rather, we argue that to effectively harness their capabilities we need to find ways to allow network management systems to explicitly control their operation so that they can coordinate across administrative boundaries.

In this paper we argued that the following requirements must be satisfied: i) the ability to deal with diversity of inter-domain controls between different providers or organizations, and ii) the ability to support various adaptive processes that may be embedded in communication devices and networks. We address these requirements through the federation of management systems that can coordinate the operation of bio-inspired management processes within and between cooperating administrative domains. We outlined a case study relating to IPTV content distribution that illustrated how a Federal Relationship Manager could be used to dynamically create and expand 
federations of service, storage and network providers in which bio-inspired processes then adaptively control control placement and distribution.

\section{Acknowledgement}

This work has been funded by Science Foundation Ireland via the "FAME" Strategic Research Cluster, grant no. 08/SRC/I1403.

\section{References}

1. URL http://www .ucmp. berkeley.edu/protista/slimemolds.html

2. Pre-Congestion Notification (PCN) architecture IETF RFC 5559. URL http://www . ietf . org/rfc/rfc5559.txt

3. Resource ReSerVation Protocol (RSVP) version 1 functional specification, IETF RFC 2205. URL http://www.ietf.org/rfc/rfc2205.txt

4. Ahn, C.W., Ramakrishna, R.S.: A Genetic Algorithm for Shortest Path Routing Problem and the Sizing of Populations. IEEE Transactions on Evolutionary Computation 6(6), 566-579 (2002)

5. Asghar, J., Le Faucheur, F., Hood, I.: Preserving video quality in iptv networks. IEEE Transactions on Broadcasting 55(2), 386-395 (2009)

6. Balasubramaniam, S., Botvich, D., Jennings, B., Davy, S., Donnelly, W., Strassner, J.: Policy-constrained bio-inspired processes for autonomic route management. Computer Networks 53(10), 1666-1682 (2009)

7. Bhatti, R., Bertino, E., Ghafoor, A.: X-FEDERATE: a Policy Engineering Framework for Federated Access Management. IEEE Transactions on Software Engineering 32(2), 330-346 (2006)

8. Bhoj, P., Singhal, S., Chutani, S.: SLA Management in Federated Eenvironments. Computer Networks 35(1), 5-24 (2001)

9. Bouabene, G., Jelger, C., Tschudin, C., Schmid, S., Keller, A., May, M.: The Autonomic Network Architecture (ANA). IEEE Journal on Selected Areas in Communications 28(1), 4-14 (2010)

10. Cabellos-Aparicio, A., Garcia, F., Domingo-Pascual, J.: A novel available bandwidth estimation and tracking algorithm. Proceedings of IEEE/IFIP Network Operations and Management Symposium (NOMS 2008) pp. 87-94 (2008)

11. Di Caro, G., Dorigo, M.: AntNet: Distributed Stigmergetic Control for Communications Networks. Journal of Artificial Intelligence Research 9, 317-365 (1998)

12. Dobson, S., Denazis, S., Fernández, A., Gaïti, D., Gelenbe, E., Massacci, F., Nixon, P., Saffre, F., Schmidt, N., Zambonelli, F.: A survey of autonomic communications. ACM Transactions on Autonomic Adaptive Systems 1, 223-259 (2006)

13. Dressler, F., Akan, O.: Bio-inspired networking: from theory to practice. Communications Magazine, IEEE 48(11), 176 -183 (2010)

14. Ekelin, S., Nilsson, M., Hartikainen, E., Johnsson, A., Mangs, J.E., Melander, B., Bjorkman, M.: Real-time measurement of end-to-end available bandwidth using kalman filtering. Proceedings of IEEE/IFIP Network Operations and Management Symposium (NOMS 2006) pp. 73-84 (2006)

15. El-Kalam, A.A., Baida, R., Balbiani, P., Benferhat, S., Cuppens, F., Deswarte, Y., Miege, A., Saurel, C., Trouessin, G.: Organization based Access Control. In: Proceedings of 4th IEEE International Workshop on Policies for Distributed Systems and Networks, pp. 120131. IEEE (2003)

16. Gogineni, H., Greenberg, A., Maltz, D., Ng, T., Yan, H., Zhang, H.: Mms: An autonomic network-layer foundation for network management. IEEE Journal on Selected Areas in Communications 28(1), $15-27$ (2010)

17. Goldoni, E., Rossi, G., Torelli, A.: Assolo, a new method for available bandwidth estimation. Proc. International Conference on Internet Monitoring and Protection pp. 130-136 (2009) 
18. Grefen, P.J., Aberer, K., Ludwig, H., Hoffner, Y.: Crossflow: Cross-organizational workflow management for service outsourcing in dynamic virtual enterprises. IEEE Data Engineering Bulletin 24(1), 52-57 (2001)

19. Hudert, S., Ludwig, H., Wirtz, G.: Negotiating SLAs - an approach for a generic negotiation framework for WS-Agreement. Journal of Grid Computing 7(2), 225-246 (2009)

20. Jain, M., Dovrolis, C.: Pathload: A measurement tool for end-to-end available bandwidth. Proceedings of Passive and Active Measurements Workshop pp. 14-25 (2002)

21. Jennings, B., Feeney, K., Brennan, R., Balasubramaniam, S., Botvich, D., van der Meer, S.: Autonomic Network Management Principles, eds. N Agoulmine and J Won-Ki Hong, chap. Federating Autonomic Network Management Systems for Flexible Control of End-to-end Communications Services, pp. 101-118. Elsevier (2010)

22. Keller, A., Ludwig, H., Kar, G., Dan, A., Hellerstein, J.L.: Managing dynamic services: A contracts-based approach to a conceptual architecture. In: Proc. 2002 IEEE/IFIP Network Operations and Management Symposium (NOMS 2002), pp. 513-528 (2002)

23. Kephart, J., Chess, D.: The vision of autonomic computing. Computer 36(1), 41-50 (2003)

24. Latré, S., De Vleeschauwer, B., Van de Meerssche, W., Perrault, S., De Turck, F., Demeester, P., De Schepper, K., Hublet, C., Rogiest, W., Custers, S., Van Leekwijck, W.: An autonomic pcn based admission control mechanism for video services in access networks. IFIP/IEEE International Symposium on Integrated Network Management (IM 2009) Workshops pp. 161-168 (2009)

25. Leibnitz, K., Wakamiya, N., Murata, M.: Biologically Inspired Self-Adaptive Multi-path Routing in Overlay Networks. Communications of the ACM 49(3), 62-67 (2006)

26. Meisel, M., Pappas, V., Zhang, L.: A taxonomy of biologically inspired research in computer networking. Computer Networks 54(6), 901-916 (2010)

27. Meskill, B., Davy, A., Jennings, B.: Server selection and admission control for ip-based video on demand using available bandwidth estimation. In: Proceedings of the 36th IEEE Conference on Local Computer Networks (LCN 2011), pp. 255-258 (2011)

28. Meskill, B., Davy, A., Jennings, B.: Revenue-maximizing server selection and admission control for IPTV content servers using available bandwidth estimates. In: Proceedings of the 2012 IEEE/IFIP Network and Operations Management Symposium (NOMS 2012), pp. 319-326. IEEE (2012)

29. Nakano, T.: Biologically inspired network systems: A review and future prospects. IEEE Transactions on Systems, Man, and Cybernetics 41(5), 630 -643 (2011)

30. Nurmela, T., Kutvonen, L.: Service Level Agreement Management in Federated Virtual Organizations. In: Distributed Applications and Interoperable Systems, LNCS 4531, pp. 62-75 (2007)

31. Paul, S., Pan, J., Jain, R.: Architectures for the future networks and the next generation internet: A survey. Computer Communications 34(1), 2 - 42 (2011)

32. Ribeiro, V.J., Riedi, R.H., Baraniuk, R.G., Navratil, J., Cottrell, L.: pathchirp: Efficient available bandwidth estimation for network paths. In: Passive and Active Measurement Workshop (2003)

33. Sommers, J., Barford, P., Willinger, W.: A proposed framework for calibration of available bandwidth estimation tools. Proc. 11th IEEE Symposium on Computers and Communications (ISCC 2006) pp. 709-718 (2006)

34. Strauss, J., Katabi, D., Kaashoek, F.: A measurement study of available bandwidth estimation tools. Proceedings of the 3rd ACM SIGCOMM conference on Internet measurement pp. 39-44 (2003)

35. Suzuki, J., Suda, T.: A Middleware Platform for a Biologically-inspired Network Architecture supporting Autonomous and Adaptive Applications. IEEE Journal on Selected Areas in Communications 23(2), 249-260 (2005)

36. Tero, A., Takagi, S., Saigusa, T., Ito, K., Bebber, D.P., Fricker, M.D., Yumiki, K., Kobayashi, R., Nakagaki, T.: Rules for Biologically Inspired Adaptive Network Design. Science 327(5964), 439-442 (2010)

37. Wang, Y., Nakao, A.: On socially-inspired cooperative and efficient overlay network evolution based on group selection pattern. In: Proceedings of the 3rd International Conference on Bio-Inspired Models of Network, Information and Computing Sytems (Bionetics 2008), p. Article No. 21 (2008)

38. Wang, Y., Nakao, A.: On cooperative and efficient overlay network evolution based on a group selection pattern. IEEE Transactions on Systems, Man, and Cybernetics, Part B: Cybernetics, 40(2), 493-504 (2010) 
39. Zieba, J., Kryza, B., Slota, R., Dutka, L., Kitowski, J.: Ontology alignment for contract based virtual organizations negotiation and operation. In: Proceedings of International Conference on Parallel Processing and Applied Mathematics, LNCS 4967, pp. 835-843 $(2007)$ 